\title{
Influence of Multiple Factors on the Wettability and Surface Free Energy of Leaf Surface
}

\author{
Yong $\mathrm{He} \oplus^{\circledR}$, Shupei Xiao, Jianjian Wu and Hui Fang * \\ College of Biosystems Engineering and Food Science, Zhejiang University, Hangzhou 310058, China; \\ yhe@zju.edu.cn (Y.H.); 180312@zju.edu.cn (S.X.); 11713011@zju.edu.cn (J.W.) \\ * Correspondence: hfang@zju.edu.cn; Tel.: +86-135-8843-3933
}

Received: 26 December 2018; Accepted: 8 February 2019; Published: 11 February 2019

\begin{abstract}
The wettability of plant leaves directly reflects leaf hydrophilicity, which is the key factor that influences the adhesion of liquid pesticide as well as affects plant protection products (PPP) efficacy. Generally, the wettability of leaf surface is quantified by the contact angle and surface free energy (SFE), which are mainly dependent on leaf surface properties, liquid properties and other spraying parameters. Therefore, the aim of this paper was to investigate the SFE of rice and rape leaves with the variation of leaf status, leaf surface, and probe liquid as well as the influence of droplet falling height, solid surface, and PPP concentration on the wettability. The results showed that: (1) the dispersive components of SFE of rice and rape account for a large proportion which are closely related to their hydrophobicity - the abaxial of rape new leaf and the adaxial of rape old leaf are easier to wet comparing with rice and rape leaves in other statuses; (2) the increase of droplet falling height had a significant effect on improving the wettability between wax surface and adjuvant solution, while it had little improving effect on the wettability between wax surface and water; (3) the wettability of different solid surface varied greatly, and the order of wettability from good to bad is water-sensitive paper (WSP), wax, rape leaf, and rice leaf; (4) the effect of PPP concentration on the leaf surface wettability is significant, the contact angle decreased with the increase of PPP concentration, and the wettability of microemulsion is better than that of suspending agent and wettable powder. In conclusion, the SFE and wettability of crop leaf surface determine the suitable type of PPP, studying the influence of multiple factors on leaf surface wettability can provide a reliable reference for providing scientific guidance as well as improving the effective utilization of PPP.
\end{abstract}

Keywords: wettability; surface free energy; contact angle; droplet falling height; plant protection products

\section{Introduction}

An efficacious and efficient spray application is an important goal to increase the benefits of plant protection products (PPP) used for crop pest and disease control [1]. The ultimate purpose of using different spray application techniques is to ensure an adequate deposition of active components of PPPs on target crops [2]. The PPPs are sprayed on the surface of the crop with a water or oil solution, and the wettability of leaf surface, adhesion force, spreading and retention behavior of PPP solution on leaf surface are the key factors affecting the exertion of PPP efficacy [3]. Generally, leaf surface wettability, which directly reflects the leaf surface hydrophilicity [4], is the result of the interaction between the surface tension of liquid and the properties of leaf surface. The leaf surface wettability affects the water absorption, gas exchange, pathogen infection, and other functions of the plant leaf [5].

Improving the effective utilization of PPPs, reducing at the same time the pesticide losses, is the main goal in the field of chemical spray application for pest and disease control [6]. The effective 
utilization rate of PPPs generally refers to the proportion of PPPs that play the role of pest and disease control in the total amount of PPPs. When applied with conventional spray technique, the effective utilization rate of PPPs is only $20-30 \%$ and only a small portion could be deposited on the target crops [7]. During the process of droplets falling on the surface of plant leaves, some droplets will adhere to the leaf surface, while some other droplets will rupture, rebound, and slip off [8]. The wettability of droplet on leaf surface is closely related to the properties of leaf surface and PPP; the formulation of PPP can be varied according to the specific crop, thus the wettability of leaf surface should be clarified initially. To specifically quantify the wettability, contact angle and surface free energy (SFE) are the most commonly used indicators [9]. Contact angle is a quick and convenient indicator to evaluate the wettability between test liquid and solid surface [10]. When the contact angle is $0^{\circ}$, the liquid completely infiltrates the solid surface; when the contact angle is $180^{\circ}$, the liquid is spherical on the solid surface and the liquid completely un-infiltrated the solid surface; when the contact angle is between $0^{\circ}$ and $90^{\circ}$, it is considered to be hydrophilic; when the contact angle is between $90^{\circ}$ and $180^{\circ}$, it is considered to be hydrophobic [11]. However, different scholars have defined different boundaries between contact angle and wetting range according to the study purpose [12]. Moreover, SFE belongs to one of the basic thermodynamic properties of solid surface and it does not change with the variation of test liquids [13]. Generally, the hydrophobic surface tends to be low energy while the hydrophilic surface tends to be high energy [14].

On one hand, some researchers have indicated that the wettability of plant leaf surface is closely related to the content and morphological distribution of wax on a leaf surface [15], density, texture, and distribution of leaf villus [16], and the number of stomata [17]. Takamatsu et al. [18] found that some hydrophobic leaves tend to have high-density wax distribution, while the wettability of the leaves with more wax content is relatively small. Zhu et al. [19] studied the wettability of rape leaf, and their results suggested that the fresh rape leaves exhibited hydrophobicity but with a high adhesion force. Besides this, Sun et al. [20] supposed that the compound structure of mastoid and waxy layer was the fundamental reason affecting the wettability of lotus leaves. The research of Lee et al. [21] pointed out that the hierarchical structures of rice leaf surfaces was the basic mechanism of rice leaf hydrophobicity. Moreover, Kumar et al. [22] found that the leaves with high villous density had strong hydrophobicity while the leaves with low villous density had good wettability. Pandey et al. [23] tested the wettability of 30 species plant leaves and suggested that the leaf with the highest stomatal density had the worst wettability.

On the other hand, since leaf surface wettability is the result of the interaction between liquid surface tension and leaf surface properties, the wettability of plant leaves is also affected by the properties of PPP as well as some external factors such as spray application technique [24] together with sprayer adjustments [25]. Xu et al. [26] suggested that the use of reasonable concentration of adjuvants could change the behavior of droplets on the leaf surface, which could improve the uniformity of spray coverage and reduce PPP use. Zyl et al. [27] found that adding adjuvants in spraying solution could increase the deposition of both sides of grape leaves and the optimum effect of droplet deposition can be achieved by matching the concentration of adjuvants with the spray application volume. Dorr et al. [28] simulated the behavior of droplet on leaf surface using a constructed virtual leaf model for chenopodium, wheat, and cotton. The results showed that the droplet characteristics, spray formulation, and leaf surface properties all affected the amount of droplet retained on the horizontal leaf.

At present, most research focused on the correlation between leaf surface properties and wettability as well as the effects of external spray parameters on droplet deposition; there are few studies that concentrated on the factors directly affecting the leaf surface wettability. Therefore, the main purpose of this study is to investigate the SFE difference of rice and rape leaves as well as the effect of multiple factors on wettability. That is, to (1) investigate the effect of crop type, leaf surface, probe liquid, and leaf status on contact angles as well as the difference of SFE and its components in rice and rape leaves; (2) investigate the effect of droplet falling height and the instantaneous velocity of droplet 
contacting solid surface on the variation of wettability; (3) investigate the variation of wettability between different solid surfaces (rice leaves, rape leaves, water-sensitive paper, and wax); and (4) investigate the influence of PPP solution concentration on wettability based on rape leaf surface.

\section{Materials and Methods}

\subsection{Experimental Materials}

\subsubsection{Solid Surface Materials}

Four types of solid surfaces were used for experimental process: water-sensitive paper (WSP), wax, rice and rape leaf; WSP represents hydrophilic surface, wax represents hydrophobic surface while the leaves of rice and rape are chosen because they are popular crops in China. The varieties of rice and rape are japonica rice Huke No. 3 and Zhejiang University No. 619, respectively. To accurately identify the phenological development platforms of plants, the Biologische Bundesanstalt, Bundessortenamt und CHemische Industrie (BBCH) scale is used [29] and the BBCH scale of rice and rape in this study were 12 and 17 , respectively.

\subsubsection{Test liquid Materials}

For evaluating the solid SFE, three probe liquids with different surface tensions were selected, which were deionized water, formamide $\left(\mathrm{CH}_{3} \mathrm{NO}, \mathrm{AR}, 99 \%\right)$, and ethylene glycol $\left(\mathrm{C}_{2} \mathrm{H}_{6} \mathrm{O}_{2}, \mathrm{AR}, 98 \%\right)$. The deionized water was made by EPED-E2-10TJ ultrapure water manufacturing system (Nanjing Eped Technology Development Co.,Ltd, Nanjing, China), formamide and ethylene glycol were from Macklin (Shanghai Macklin Biochemical Co., Ltd, Shanghai, China). The surface properties of these probe liquids are listed in Table 1 [30-32].

Table 1. Probe liquid for surface free energy (SFE) calculation and their surface tension.

\begin{tabular}{cccc}
\hline Probe Liquids & $\begin{array}{c}\text { Total Surface } \\
\text { Tension }(\mathbf{m N} / \mathbf{m})\end{array}$ & $\begin{array}{c}\text { Polar Component } \\
(\mathbf{m N} / \mathbf{m})\end{array}$ & $\begin{array}{c}\text { Dispersive Component } \\
\mathbf{( m N / m )}\end{array}$ \\
\hline deionized water & 72.80 & 43.70 & 29.10 \\
formamide & 58.20 & 23.10 & 35.10 \\
ethylene glycol & 48.20 & 18.91 & 29.29 \\
\hline
\end{tabular}

In this experiment, one adjuvant and five common PPP for rice and rape were used to investigate the multiple factors on leaf surface wettability. All the PPP mentioned in Table 2 were from the Institute of Plant Protection (Chinese Academy of Agricultural Sciences, Beijing, China). According to agrochemical label recommended dosage, the concentration prepared is shown in Table 2.

Table 2. Reagent name, formulations, and concentrations of plant protection products (PPP).

\begin{tabular}{ccc}
\hline Reagent Name & Formulation & Concentration \\
\hline organosilicone & adjuvant & $0.33 \mathrm{~g} / \mathrm{L}$ \\
prochloraz & $30 \%$ microemulsion & $2.67 \mathrm{~g} / \mathrm{L}$ \\
pymetrozine & $25 \%$ suspending agent & $2 \mathrm{~g} / \mathrm{L}$ \\
tricyclazole & $20 \%$ wettable powder & $3.33 \mathrm{~g} / \mathrm{L}, 10 \mathrm{~g} / \mathrm{L}-100 \mathrm{~g} / \mathrm{L}, 10 \mathrm{~g} / \mathrm{L}$ per gradient \\
dimethachlon & $40 \%$ wettable powder & $1 \mathrm{~g} / \mathrm{L}, 5 \mathrm{~g} / \mathrm{L}, 10 \mathrm{~g} / \mathrm{L}-50 \mathrm{~g} / \mathrm{L}, 10 \mathrm{~g} / \mathrm{L}$ per gradient \\
iprodione & $25 \%$ suspending agent & $2 \mathrm{~g} / \mathrm{L}, 10 \mathrm{~g} / \mathrm{L}-100 \mathrm{~g} / \mathrm{L}, 10 \mathrm{~g} / \mathrm{L}$ per gradient \\
\hline
\end{tabular}

\subsection{Experimental Instrument}

The experimental instrument was as follows: the contact angle measuring device (Figure 1c) Dataphysics OCA 50AF (Data Physics Instruments GmbH, Germany) was used to measure the contact angle between test liquid and solid surfaces, which was capable of auto focusing with 6 times zoom 
lens and the measuring accuracy is $\pm 0.1^{\circ}$. The halogen lamp can be continuously adjusted with matching control software and the sample table can be adjusted in XYZ three directions. Ultra-high resolution scanning electron microscope HITACHI SU8010 (Hitachi Ltd., Japan) was used to obtain the scanning electron microscope (SEM) photos of rape and rice leaves. This instrument has high resolution with the highest resolution of $1.1 \mathrm{~nm}$ in $1 \mathrm{kV}$ deceleration mode and $0.8 \mathrm{~nm}$ in $15 \mathrm{kV}$ mode. In addition, in order to investigate the effect of droplet falling height (instantaneous velocity of droplet contacting solid surface) on contact angle, a high-speed camera (Figure 1a) pco.dimax HD (PCO.Imaging, Germany) was used to record the complete process of droplet falling. This camera has 2.8 million pixels and ultra-high sensitivity, the maximum camera shooting speed is $1603 \mathrm{fps}$ at full resolution $(1920 \times 1440$ pixels) and $3822 \mathrm{fps}$ at $1008 \times 1000$ pixels, the shortest shutter time is $1.5 \mu \mathrm{s}$.

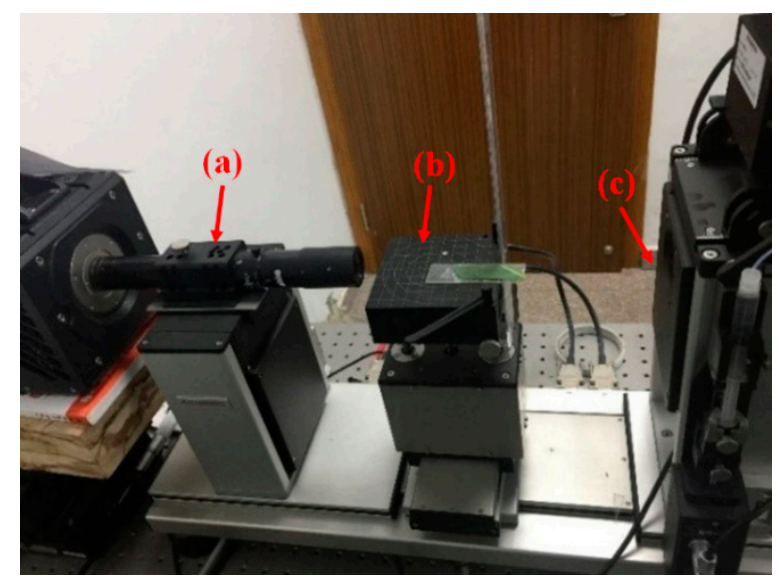

Figure 1. Contact angle measuring device: high speed camera (a), sample platform (b) and contact angle measuring device (c).

\subsection{Experimental Methods}

\subsubsection{Contact Angle Determination}

Figure 2 shows the static contact angel $(\theta)$ of droplet on solid surface, which is an important index to quantitatively evaluate the wettability of solid surface [33]. The clean WSP and wax were directly placed on the sample platform, while the leaves were cut into pieces (avoiding veins and lesions) and fixed on the glass slide. Dataphysics OCA 50AF was used to measure contact angle, and the contact angle measurement was taken immediately after the droplet was placed on the solid surface by a $1 \mu \mathrm{L}$ pipettor. In individual contact angle measurements between each test liquid and each solid surface, each contact angle was measured 5 times and 10 measurement points were selected.
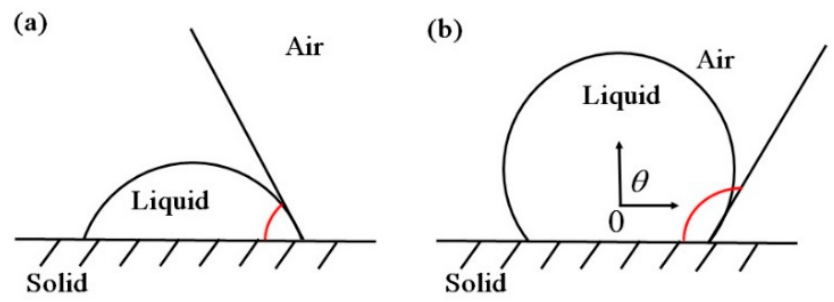

Figure 2. Static contact angel $(\theta)$ of droplet on solid surface: (a) droplet performs high wettability on solid surfaces; (b) droplet performs low wettability on solid surfaces.

\subsubsection{SEM Images Acquisition}

According to the SEM sample preparation method, the SEM samples of rice and rape leaves were prepared respectively. The following procedure was applied: (a) the samples were fixed with $2.5 \%$ glutaraldehyde overnight at $4{ }^{\circ} \mathrm{C}$; (b) the samples were rinsed with phosphoric buffer $(0.1 \mathrm{M}, \mathrm{pH} 7.0)$ 
three times, 15 min each time; (c) the samples were fixed with $1 \%$ osmic acid in the ventilated cabinet with for $1.5 \mathrm{~h}$; (d) the samples were rinsed with phosphoric buffer $(0.1 \mathrm{M}, \mathrm{pH} 7.0)$ three times, $15 \mathrm{~min}$ each time; (e) the samples were dehydrated with 30\%, 50\%,70\%,80\%, $90 \%$, and $95 \%$ ethanol orderly for 15 min each time and dehydrated with absolute ethyl alcohol for $20 \mathrm{~min}$; (f) finally, the samples were dried by $\mathrm{CO}^{2}$ critical point dryer and coated, then trimmed to the appropriate size and then observed under ultra-high resolution scanning electron microscope. In this experiment, the SEM images of rape and rice leaves were obtained at the magnification times of 100 times, 300 times, 1000 times, and 5000 times respectively.

\subsubsection{Surface Free Energy Evaluation}

In order to quantify the surface properties of solid, the Owens-Wendt-Rabel-Kaelble (OWRK) method could be used to calculate the solid SFE by measuring several contact angles between test liquid and solid surface [34]. Generally, the solid SFE of each phase is divided into polar component $\gamma^{p}$ and dispersive component $\gamma^{d}$. The relationship is as follows:

$$
\gamma_{s l}=\gamma_{l}+\gamma_{s}-2\left(\gamma_{l}^{d} \gamma_{s}^{d}\right)^{1 / 2}-2\left(\gamma_{l}^{p} \gamma_{s}^{p}\right)^{1 / 2}
$$

where $\gamma_{l}^{d}$ and $\gamma_{s}^{d}$ are the dispersive component of free energy of liquid and solid, respectively, $\gamma_{l}^{p}$ and $\gamma_{s}^{p}$ are the polar component of free energy of liquid and solid, respectively.

Besides this, Young's equation, also known as the wetting equation, is the description of the relationship between interfacial tension of solid-air interface, solid-liquid interface, liquid-air interface, and contact angle [35]. When the liquid contacts the solid surface in the gaseous phase, the relationship among contact angle $\theta$, liquid surface tension $\gamma_{l}$, SFE between solid-liquid phase $\gamma_{s l}$ and solid SFE $\gamma_{s}$ is expressed as:

$$
\gamma_{s}=\gamma_{s l}+\gamma_{l} \cdot \cos \theta
$$

Substituting Equation (2) into Equation (1) gives:

$$
\gamma_{l} \cdot(1+\cos \theta)=2\left(\gamma_{l}^{d} \gamma_{s}^{d}\right)^{1 / 2}+2\left(\gamma_{l}^{p} \gamma_{s}^{p}\right)^{1 / 2}
$$

Therefore, when the contact angle between two or more kinds of liquid on the same solid surface (known $\gamma_{l}, \gamma_{l}^{d}, \gamma_{l}^{p}$ ) is obtained, the OWRK method can be used to calculate the solid SFE as well as its corresponding polar and dispersive components [13].

\subsubsection{Experimental Process}

In this paper, the experiments mainly includes four parts.

Experiment I: The SFE and its components of rice and rape leaves was evaluated. The contact angles of three probe liquids (deionized water, formamide, and ethylene glycol) on both the adaxial and abaxial surfaces of rice and rape leaves were measured, and the leaf status of rape (sixth leaf of rape and first leaf of rape) was compared as well. Each contact angle was measured 5 times and 10 measurement points were selected respectively. The SFE of rice and rape leaves as well as its polar component and dispersive component was calculated using OWRK method.

Experiment II: The effects of droplet falling height as well as the instantaneous velocity of droplet contacting solid surface on the wettability were investigated. To avoid the influence of leaf surface roughness on the experiment, a wax with smooth surface was chosen and placed on the sample platform of contact angle measuring device and the dividing ruler was fixed to the side of the sample platform. A $1 \mu \mathrm{L}$ droplet of respective water and organosilicon adjuvant solution was released from $1 \mathrm{~cm}$ height and the contact angle was measured after droplet stabilization. Each measurement was repeated 5 times at each height with the gradient of $1 \mathrm{~cm}$. In addition, the whole process of droplet falling was recorded by high speed camera at $2000 \mathrm{fps}$ rate to calculate the instantaneous velocity of 
droplet contacting solid surface. Generally, the process from droplet falling to its stationary state on the solid surface can be divided into five platforms (falling, spreading, maximum spreading, recoiling, and retain), which are shown in Figure 3.

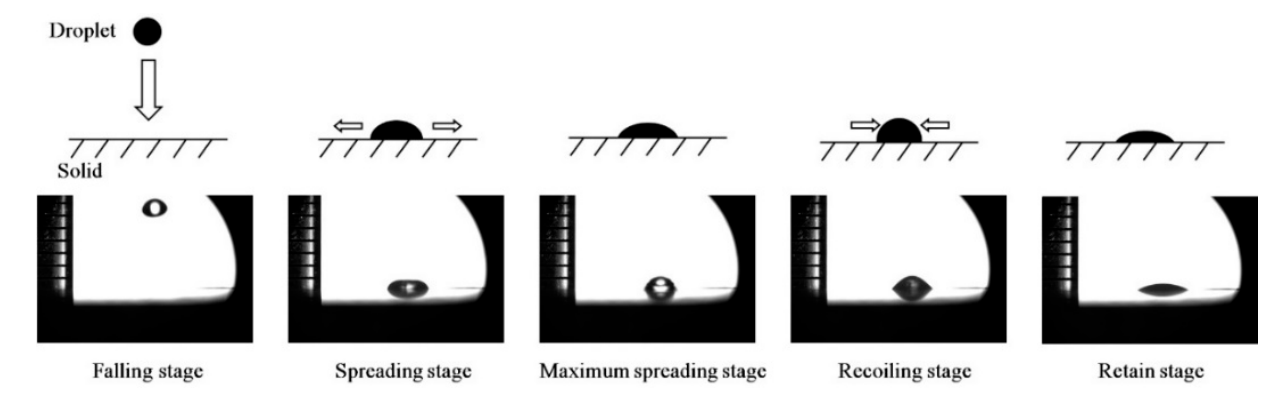

Figure 3. The collision process of between falling droplet and solid surface in five platforms.

Experiment III: The difference of wettability between the four different solid surfaces, defined above in Section 2.1.1, was explored. The new leaves were cut off from rape and rice plant and then fixed on the glass slide for the contact surface measurement. Besides this, a $1 \mu \mathrm{L}$ pipettor was used to pipet and release the droplet to the solid surface and the contact angle was measured after the droplet stabilization. Each contact angle between liquid and solid surface was measured 5 times and 10 measurement points were selected for each solid surface.

Experiment IV: The effect of the PPP concentration on the wettability of rape new leaves was investigated. In this experiment, three kinds of PPP ( $20 \%$ wettable powder tricyclazole, $40 \%$ wettable powder dimethachlon, $25 \%$ suspending agent iprodione) were used and the concentration gradient was divided in Table 2. Additionally, the new rape leaves were chosen as solid surface and the droplet was released by a $1 \mu \mathrm{L}$ pipettor. Each contact angle between liquid and solid surface was measured 5 times and 10 measurement points were selected for each test liquid.

\subsubsection{Statistical Analysis}

In this paper, to analyze the effect of crop type, rape leaf status, leaf surface, and probe liquid on contact angles, three-way analysis of variance (ANOVA) was performed by IBM SPSS Statistics 22 (International Business Machines Corporation, Armonk, NY, USA, 2013). P-value was used as statistical significance, where $\mathrm{P}>0.05$ means no significant difference, $\mathrm{P}<0.05$ means significant difference, and $\mathrm{P}<0.01$ means extreme significant difference.

\section{Results and Discussion}

\subsection{The Comparison of Surface Free Energy on Rice and Rape Leaf}

The measurements of probe liquids on rice and rape leaves both on the adaxial and abaxial surfaces are given in Table 3. When deionized water, formamide, and ethylene glycol were used as test liquids, respectively, the contact angles on rice and leaf surface showed the same variation tendency. The contact angles of both formamide and ethylene glycol were smaller than that of deionized water due to a lower surface tension. In addition, the contact angle of rice adaxial surface was higher than that of the abaxial surface, which is consistent with the new leaf of rape, while the contact angle of the adaxial surface of rape old leaf was lower than that of the abaxial surface. 
Table 3. Contact angles of probe liquids on rice and rape leaves.

\begin{tabular}{ccccc}
\hline Crop & Leaf Surface & Deionized Water $\left({ }^{\circ}\right)$ & Formamide $\left(^{\circ}\right)$ & Ethylene Glycol $\left(^{\circ}\right)$ \\
\hline rice & adaxial & $134.01 \pm 3.22$ & $126.53 \pm 2.26$ & $122.98 \pm 2.79$ \\
sixth leaf of rape & abaxial & $128.88 \pm 2.08$ & $126.30 \pm 1.39$ & $123.67 \pm 3.15$ \\
(new leaf) & adaxial & $121.97 \pm 3.59$ & $110.74 \pm 3.73$ & $103.90 \pm 4.59$ \\
first leaf of rape & abaxial & $115.57 \pm 3.66$ & $105.04 \pm 2.28$ & $96.78 \pm 3.79$ \\
(old leaf) & abaxial & $113.55 \pm 2.79$ & $104.73 \pm 1.29$ & $86.95 \pm 2.19$ \\
\hline & $122.30 \pm 3.71$ & $117.21 \pm 1.30$ & $104.69 \pm 3.74$ \\
\hline
\end{tabular}

The results of the effect of crop type, rape leaf status, leaf surface, and probe liquid on contact angles using ANOVA were given in Tables 4 and 5. We found that the crop type (rice and rape), leaf surface (adaxial and abaxial surface), and probe liquids (deionized water, formamide, and ethylene glycol) have significant difference $(\mathrm{P}<0.001)$ on contact angles. While there was no practical difference between the contact angles and rape leaf status $(P=0.416)$, indicating that the difference between new leaf and old leaf of rape had less influence on leaf surface wettability compared with the factors of crop type, leaf surface, and probe liquid, and the influence of probe liquid could also be found in Gao's research [3].

Table 4. Effect of crop type, leaf surface, and probe liquid on contact angles.

\begin{tabular}{cccccc}
\hline Source & $\begin{array}{c}\text { Type III Sum } \\
\text { of Squares }\end{array}$ & DF & MS & F & P-Value \\
\hline crop type & 4893.393 & 1 & 4893.393 & 390.432 & $<0.001$ \\
leaf surface ${ }^{1}$ & 238.052 & 1 & 238.052 & 18.994 & $<0.001$ \\
probe liquid & 1784.951 & 2 & 892.476 & 71.209 & $<0.001$ \\
\hline
\end{tabular}

${ }^{1}$ The adaxial and abaxial surface; DF: degrees of freedom; MS: mean square; F: test statistics.

Table 5. Effect of rape leaf, leaf surface, and probe liquid on contact angles.

\begin{tabular}{cccccc}
\hline Source & $\begin{array}{c}\text { Type III Sum } \\
\text { of Squares }\end{array}$ & DF & MS & F & P-Value \\
\hline rape leaf ${ }^{1}$ & 8.723 & 1 & 8.723 & 0.672 & 0.416 \\
leaf surface & 162.368 & 1 & 162.368 & 12.512 & 0.001 \\
probe liquid & 4127.167 & 2 & 2063.583 & 159.020 & $<0.001$ \\
\hline \multicolumn{4}{c}{ The sixth leaf of rape (new leaf) and the first leaf of rape (old leaf). }
\end{tabular}

The SFE of rice and rape leaves as well as its dispersive and polar components was calculated based on the OWRK method. In general, the SFE of $0-20 \mathrm{~mJ} / \mathrm{m}^{2}$ is considered to be highly hydrophobic while the SFE of $20-40 \mathrm{~mJ} / \mathrm{m}^{2}$ is considered to be hydrophobic [3]. The surface shows hydrophilicity when the polar component is dominant, while it shows hydrophobicity when the dispersive component is dominant [36]. According to Figure 3, all of the tested leaves of rice and rape (two growth platforms), in two statuses for each, adaxial and abaxial, performed with high hydrophobicity $\left(\right.$ SFE $<20 \mathrm{~mJ} / \mathrm{m}^{2}$ ) from the perspective of SFE value. The dispersion components of all leave statuses accounted for a larger proportion than polar component. Except for the abaxial surface of rape old leaves $(68.50 \%)$, the dispersive components of the remaining rice and leaves accounted for more than $90 \%$. Besides this, there was a great difference in the SFE between rice and rape leaves, where the hydrophobicity of rice leaves is stronger than that of rape leaves based on the values of contact angle and SFE. On one hand, the SFE of rape new leaves is higher than that of old leaves, and the SFE of the adaxial surface is a little lower than that of the abaxial surface in rape new leaves while the SFE of the adaxial surface is much higher than that of the abaxial surface in rape old leaves. It is indicated that the wettability of adaxial surface is generally better than that of the abaxial surface, except for the decaying leaves, which is correlated with the stomatal distribution of the leaf [17]. On the other hand, the SFE of the adaxial 
surface of rice leaves is slightly higher than that of the abaxial surface, and the dispersive component accounts for the larger proportion in the adaxial surface, which suggests that the wettability difference of the adaxial and abaxial surfaces of rice leaves is smaller comparing to rape leaves, which could also be confirmed in Figure 4.

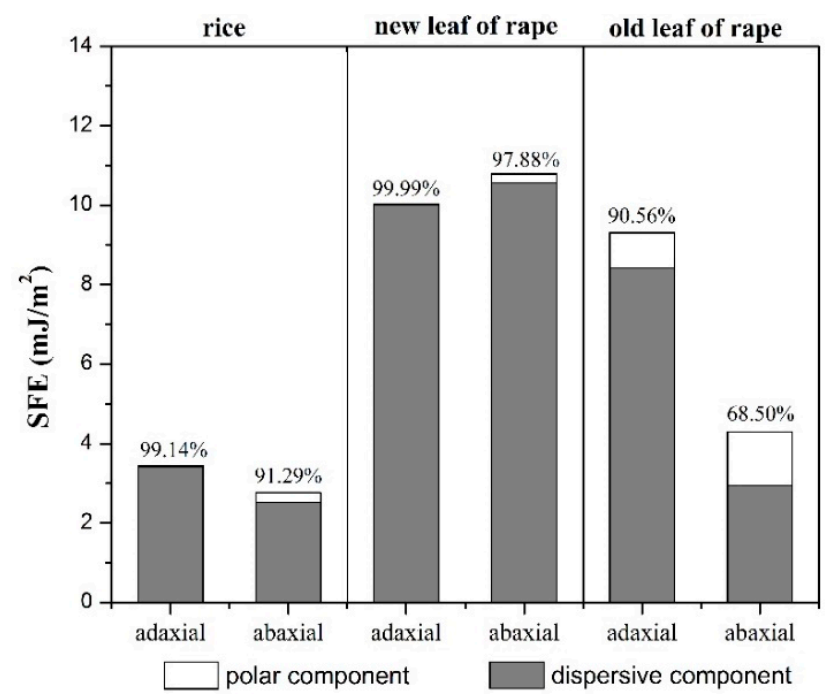

Figure 4. Surface free energy and its components of rice leaf and rape leaf on both adaxial and abaxial surfaces (\% represents the ratio of dispersive component to the SFE).

\subsection{The Influence of Droplet Falling Height on Wettability}

Figure 5 shows six partial screenshots of droplets falling from $1 \mathrm{~cm}$ altitude. Among them, (a) and (d) refer to the first frame that the droplet (water and organosilicon adjuvant) appears in the lens field of vision; (b) and (e) refer to the moment immediately before the droplet touches the solid surface; (c) and (f) refer to stable status of droplets on the solid surface. Based on the number of shooting frames of the high-speed camera and the corresponding time difference, it is possible to calculate the instantaneous velocity of the droplet touching the wax surface.

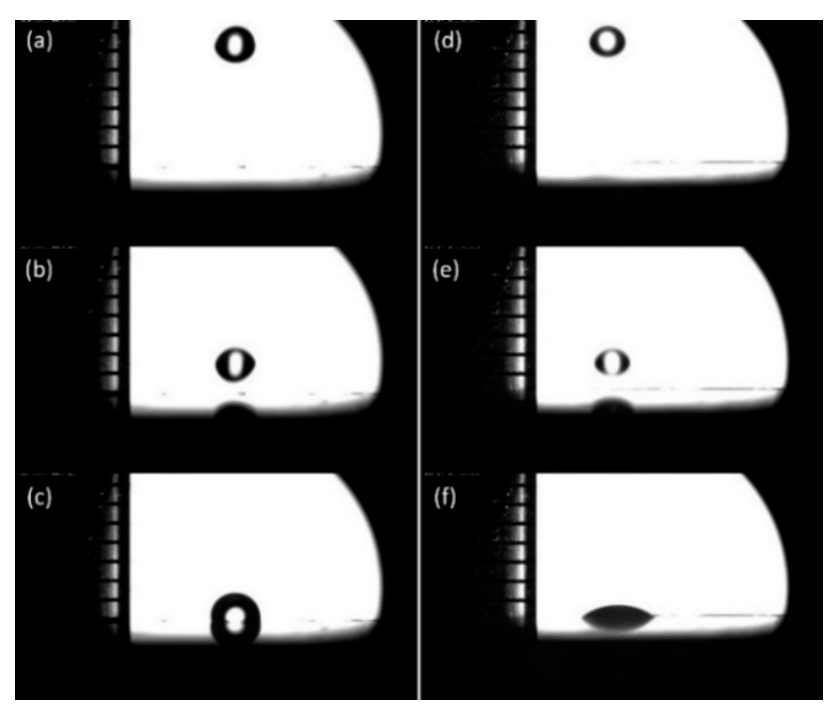

Figure 5. Partial screenshots of droplets falling from $1 \mathrm{~cm}$ altitude: water droplet $(\mathbf{a}, \mathbf{b}, \mathbf{c})$ and organosilicon adjuvant droplet $(\mathbf{d}, \mathbf{e}, \mathbf{f})$.

Figure 6 shows the variation of contact angle of droplet on the wax surface with the increase of droplet falling height. When the water droplets were falling, the contact angle fluctuated between $97^{\circ}$ 
and $103^{\circ}$ with the increase of droplet falling height. It indicates that there is an obvious hydrophobic relationship between the wax and water droplet, and the increase of the droplet falling height as well as the instantons velocity of the droplet contacting wax surface does not change the hydrophilicity between the droplet and the wax surface. However, when the organosilicon droplets were falling, the contact angle between droplet and wax surface was only $34.04^{\circ}$ at $1 \mathrm{~cm}$ droplet falling height. The contact angle between organosilicon droplet and wax was obviously better than that of the water droplet, which indicates that the organosilicon adjuvant could improve the wettability to a large extent. Besides this, the contact angle was only $17.38^{\circ}$ when the droplet falling height was increased to $2 \mathrm{~cm}$. It tended to be stable with the increase of droplet falling height, and finally fluctuated between $12^{\circ}$ and $13^{\circ}$. Therefore, it can be seen that the use of organosilicon adjuvant can greatly increase the surface tension of droplets. Since the organosilicon droplets were already extremely hydrophilic at low falling altitudes, the variation of contact angle was small as the falling height rose continuously, where the contact angle tended to be stable gradually.

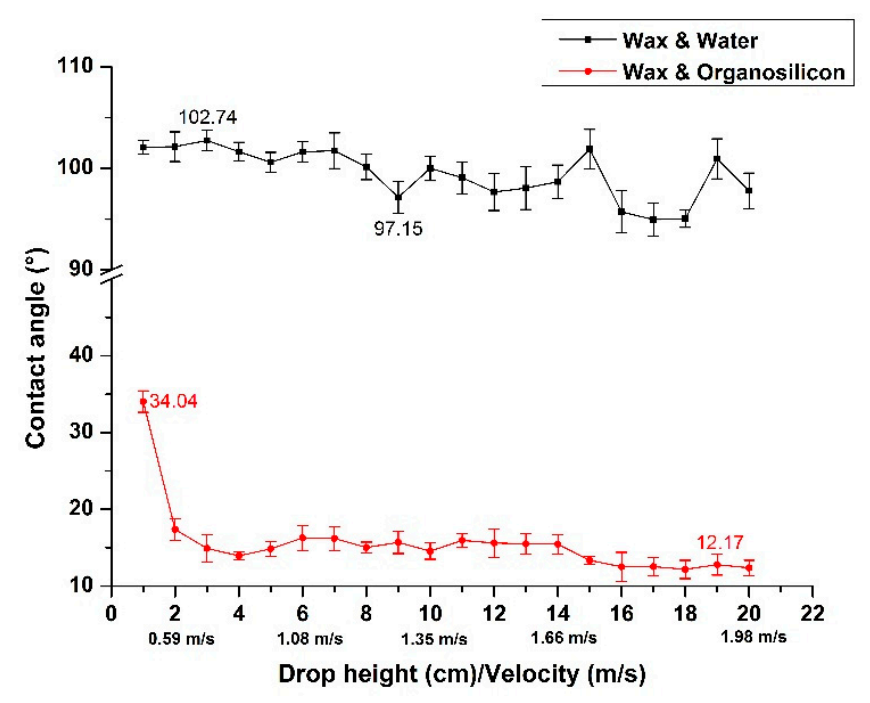

Figure 6. The variation of wettability with the increase of droplet falling height.

\subsection{The Influence of Solid Surface on Wettability}

In order to investigate the effect of solid surface on wettability, four types of solid surfaces and four kinds of reagents were selected as solid surface and test liquid, respectively, which has been described in detailed in Section 2.1. Figure 7 shows the SEM images of rice and rape leaves at 1000 and 5000 magnification times, respectively. 


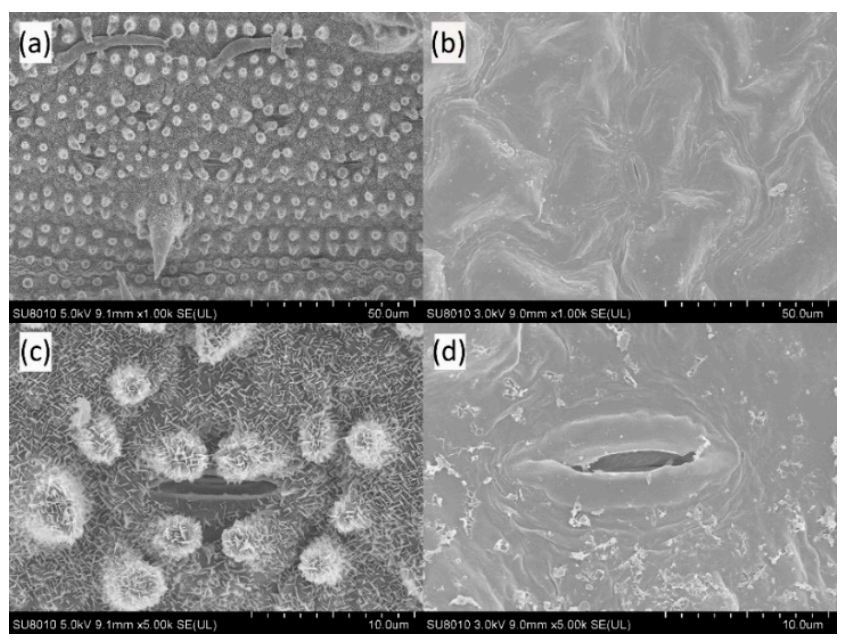

Figure 7. High magnification SEM of rape and rice leaves (adaxial surface): (a) rice of 1000 times; (b) rape of 1000 times; (c) rice of 5000 times; (d) rape of 5000 times.

The non-smooth surface structure of most plant leaves, such as furs, spines, protrusions, and other appendages, is a direct factor affecting the hydrophobicity of leaves [37]. From the view of the microstructure of leaves, the surface of rice leaves is rough with a regular arrangement of beaded microprotrusions (Figure 5a), whose average diameter is around 5-8 $\mu \mathrm{m}$ [38]. Some studies showed that those microprotrusions are the mixture of wax on the leaf surface and silicon oxide with strong hydrophobicity [39], while the leaf surface is covered with dense acerose arrangement at the magnification of 5000 times (Figure 5c). In addition, the surface of rape leaves is relatively smooth and the waxy morphology of the leaves is irregularly arranged with the shape of blocks. The wax around the stomata is in the form of small rods [40], and there are filamentous appendages around the stomata (Figure 5d). In general, some results in current research indicated that the wettability of leaf surface with less waxiness and sparse villus distribution is better.

Figure 8 compares the wettability of four solid surfaces tested with different PPP. It can be seen that the hydrophilicity of four solid surface from high to low is WSP, wax, rape leaf, and rice leaf. Specifically, when using water for experiment, only the contact angle with WSP was less than $90^{\circ}$ and appeared as hydrophilic, while the water droplet with rice leave, rape leave, and wax appeared as hydrophobic. Besides this, the wettability of rice leaves changed from hydrophobic to hydrophilic while using three PPP to replace water droplet, among which the wettability of rice leaves was greatly improved by using pymetrozine and decreasing the contact angle to about $80^{\circ}$.

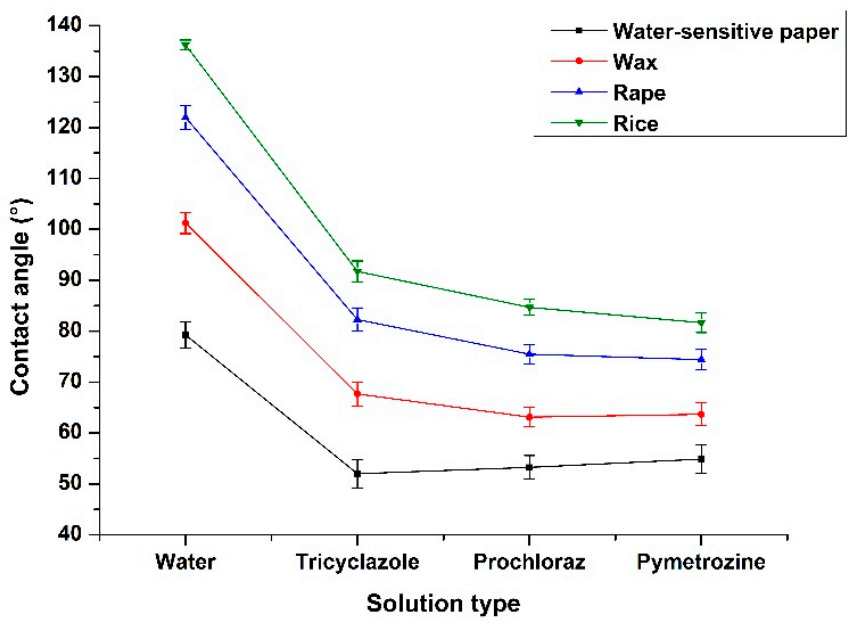

Figure 8. The variation of contact angle with the variation of solid surfaces and PPP. 
For the influence of PPP types on the wettability of solid surface, the contact angle between WSP and three kinds of PPP was from $50^{\circ}$ to $60^{\circ}$. The results of wettability between rice, rape, and wax on the three PPP showed that the contact angle of $25 \%$ suspending agent pymetrozine was slightly lower than that of $30 \%$ microemulsion prochloraz, and both of them were obviously superior to $20 \%$ wettable powder tricyclazole. It is concluded that the solubility of tricyclazole as a wettable powder is poor, whose solution is a mixture of powder and water and there is sediment in the solution after resting. Thus, the effect of improving wettability is not as good as that of suspending agent and microemulsion.

\subsection{The Influence of PPP Concentration on Wettability}

In order to investigate the effect of the PPP concentration on the wettability of rape leaves, $20 \%$ wettable powder tricyclazole, $40 \%$ wettable powder dimethachlon, and $25 \%$ suspending agent iprodione were divided into different concentration gradients according to the agrochemical label recommended dosage. The contact angle varies with the concentration of three PPPs was shown in Figure 9.

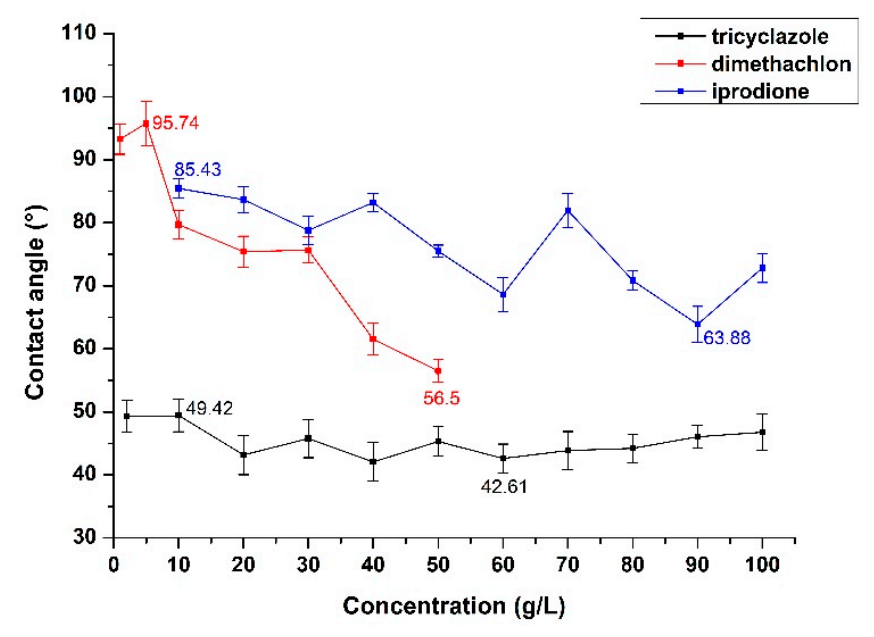

Figure 9. The variation of contact angle with the variation of PPP concentration.

Wettable powder is one of the earliest and most commonly used PPP reagents, while the biggest problem while using is that the poor solubility of the wettable powder results in the precipitate of the particles. Thus, the wettable powder solution needs to be stirred frequently during the spraying process to ensure its uniformity. Comparing to wettable powder, the particle size of suspending agent is smaller and the suspension rate is higher, thus it is not easy to precipitate and the stability of suspending agent is better as well. In this experiment, three kinds of PPP with different formulation were used.

According to Figure 9, tricyclazole is a kind of protective fungicide with strong internal absorption, whose contact angle fluctuates between $42^{\circ}$ and $50^{\circ}$ along with a slow decreasing trend as the concentration increases. In general, the variation of contact angle with tricyclazole concentration is almost negligible and a possible explanation is that the solubility of $20 \%$ wettable powder tricyclazole is poor, as a lot of sediments were found in the bottom of test tube. Although the shaking treatment was done before the test, the effective components of tricyclazole in the solution may have reached the saturation degree. In addition, dimethachlon is a potent fungicide, which is the most commonly used towards rape among three PPP. Dimethachlon showed the largest decrease in contact angle with the increase of dimethachlon concentration, and the wettability of rape leaves was almost not improved when the concentration was at a low level, which was close to the contact angle between water and rape leaves $\left(96.56^{\circ}\right)$. With the continuous increase of dimethachlon concentration, the wettability of rape leaves increased significantly and the contact angle decreased to about $80^{\circ}$ when the concentration reached $10 \mathrm{~g} / \mathrm{L}$. Besides this, iprodione is a kind of contact fungicide with broad range and high 
efficiency, whose contact angle decreases gradually with the increase of iprodione concentration and it also fluctuates in a wide range between $63^{\circ}$ and $86^{\circ}$. The reason might be that the solution has reached its saturated state when the concentration is high, and the bubble and sediment in the solution might also affect the measurement of contact angle to some extent. In general, with the increase of PPP concentration, the wettability increased differently depending on the formulation of PPP, and the wettability of microemulsion is better than that of suspending agent and wettable powder.

\section{Conclusions}

In this study, the difference of SFE and its components was investigated on rice and rape leaves, and the effects of droplet falling height, solid surface, and PPP concentration on wettability were studied. Contact angle and SFE were used to characterize the variation of wettability. Based on the results and analysis, the following conclusions can be obtained.

(1) The crop type, leaf surface, and probe liquid have significant differences on the variation of contact angles. There was no practical difference between the contact angles and rape leaf status (new leaf and old leaf).

(2) The enhancement of droplet falling height and the reasonable selection of PPP can improve the solid surface wettability to a certain extent.

(3) The hydrophilicity of the four types of solid surfaces from high to low is WSP, wax, rape leaf, then rice leaf, and the wettability significantly improved when the farm chemicals were used instead of water.

(4) In the range of solution saturation, the contact angle decreases with different degrees according to reagent type with the increase of PPP solution concentration. Generally, the wettability of microemulsion is better than that of suspending agent and wettable powder.

Author Contributions: Y.H. and S.X. conceived and designed the experiments; S.X. and J.W. performed the experiments; Y.H., S.X., and H.F. analyzed the data; Yong He wrote the drift manuscript; S.X., J.W., and H.F. helped revise the manuscript.

Funding: This research was funded by The National Key Research and Development Program of China, grant number 2016YFD0200701.

Conflicts of Interest: The authors declare no conflict of interest.

\section{References}

1. Grella, M.; Gallart, M.; Marucco, P.; Balsari, P.; Gil, E. Ground Deposition and Airborne Spray Drift Assessment in Vineyard and Orchard: The Influence of Environmental Variables and Sprayer Settings. Sustainability 2017, 9, 728. [CrossRef]

2. Xiang, D.; Zhu, H.P.; Yang, X.J. Characterization of droplet impact and deposit formation on leaf surfaces. Pest Manag. Sci. 2015, 71, 302-308.

3. Yue, G.; Guo, R.F.; Fan, R.J.; Liu, Z.F.; Kong, W.N.; Zhang, P.J.; Du, F.P. Wettability of pear leaves from three regions characterized at different stages after flowering using the OWRK method. Pest Manag. Sci. 2018, 74, 1804-1809.

4. Shi, H.; Wang, H.X.; Li, Y.Y. Wettability on plant leaf surfaces and its ecological significance. Acta Ecol. Sinica 2011, 31, 4287-4298.

5. Aryal, B.; Neuner, G. Leaf wettability decreases along an extreme altitudinal gradient. Oecologia 2010, 162, 1-9. [CrossRef] [PubMed]

6. Miranda-Fuentes, A.; Marucco, P.; González-Sánchez, E.J.; Gil, E.; Grella, M.; Balsari, P. Developing strategies to reduce spray drift in pneumatic spraying in vineyards: Assessment of the parameters affecting droplet size in pneumatic spraying. Sci. Total Environ. 2018, 616-617, 805-815. [CrossRef] [PubMed]

7. Yuan, H.; Yang, D.B.; Yan, X.; Zhang, L. Pesticide efficiency and the way to optimize the spray application. Plant Prot. 2011, 37, 14-20. 
8. Dorr, G.J.; Wang, S.S.; Mayo, L.C.; Mccue, S.W.; Forster, W.A.; Hanan, J.; He, X.K. Impaction of spray droplets on leaves: Influence of formulation and leaf character on shatter, bounce and adhesion. Exp. Fluids 2015, 56, 1-17. [CrossRef]

9. Chiou, C.H.; Hsieh, S.J. Empirical study and prediction of contact angle and surface free energy of commonly used plastics with pillar-like structure. Surf. Interface Anal. 2015, 47, 45-55. [CrossRef]

10. Benli, B.; Hao, D.; Celik, M.S. The anisotropic characteristics of natural fibrous sepiolite as revealed by contact angle, surface free energy, AFM and molecular dynamics simulation. Colloids Surfaces A 2012, 408, 22-31. [CrossRef]

11. Lin, H.; Zhou, H.; Xu, L.; Zhu, H.; Huang, H. Effect of surfactant concentration on the spreading properties of pesticide droplets on Eucalyptus leaves. Biosyst. Eng. 2016, 143, 42-49. [CrossRef]

12. Bhushan, B.; Yong, C.J. Natural and biomimetic artificial surfaces for superhydrophobicity, self-cleaning, low adhesion, and drag reduction. Prog. Mater. Sci. 2011, 56, 1-108. [CrossRef]

13. Liu, D.D.; Xu, Z.C.; Zhang, L.; Luo, L.; Zhang, L.; Wei, T.X.; Zhao, S. Adsorption behaviors of cationic surfactants and wettability in polytetrafluoroethylene-solution-air systems. Langmuir 2012, 28, 16845-16854. [CrossRef] [PubMed]

14. Nairn, J.J.; Forster, W.A. Methods for evaluating leaf surface free energy and polarity having accounted for surface roughness. Pest Manag. Sci. 2017, 73, 1854-1865. [CrossRef] [PubMed]

15. Taylor, P. The wetting of leaf surfaces. Curr. Opin. Colloid Interface Sci. 2011, 16, 326-334. [CrossRef]

16. Neinhuis, C.; Barthlott, W. Characterization and Distribution of Water-repellent, Self-cleaning Plant Surfaces. Ann. Bot. 1997, 79, 667-677. [CrossRef]

17. Brewer, C.; Nunez, C. Patterns of leaf wettability along an extreme moisture gradient in western Patagonia, Argentina. Int. J. Plant Sci. 2007, 168, 555-562. [CrossRef]

18. Takamatsu, T.; Sase, H.; Takada, J.; Matsushita, R. Annual Changes in Some Physiological Properties of Cryptomeria Japonica Leaves from Kanto, Japan. Water Air Soil Pollut. 2001, 130, 941-946. [CrossRef]

19. Zhu, H.; Guo, Z. Wetting Characterizations of Oilseed Rapes. J. Bionic Eng. 2016, 13, 213-219. [CrossRef]

20. Sun, Y.H.; Yang, X.D.; Yang, Z.J.; Wang, Q.C.; Men, Y.Z. Difference in wettability of lotus leaves in typical states and its mechanism analysis. Trans. Chin. Soc. Agric. Eng. 2014, 30, 263-267.

21. Lee, S.G.; Lim, H.S.; Dong, Y.L.; Kwak, D.; Cho, K. Tunable Anisotropic Wettability of Rice Leaf-Like Wavy Surfaces. Adv. Funct. Mater. 2013, 23, 526. [CrossRef]

22. Kumar, N.; Pandey, S.; Bhattacharya, A.; Ahuja, P.S. Do leaf surface characteristics affect Agrobacterium infection in tea [Camellia sinensis(L.) O Kuntze]? J. Biosci. 2004, 29, 309-317. [CrossRef] [PubMed]

23. Pandey, S.; Nagar, P.K. Patterns of leaf surface wetness in some important medicinal and aromatic plants of Western Himalaya. Flora 2003, 198, 349-357. [CrossRef]

24. Xu, G.C.; Gu, Z.Y.; Xu, D.J.; Xu, X.L.; Dong, Y.X. Wettability Analysis of Pesticides on Rice Leaf. Sci. Agric. Sinica 2012, 45, 1731-1740.

25. Dekeyser, D.; Foqué, D.; Duga, A.T.; Verboven, P.; Hendrickx, N.; Nuyttens, D. Spray deposition assessment using different application techniques in artificial orchard trees. Crop Prot. 2014, 64, 187-197. [CrossRef]

26. Xu, L.Y.; Zhu, H.P.; Ozkan, H.E.; Bagley, W.E.; Krause, C.R. Droplet evaporation and spread on waxy and hairy leaves associated with type and concentration of adjuvants. Pest Manag. Sci. 2011, 67, 842-851. [CrossRef] [PubMed]

27. Zyl, S.A.V.; Brink, J.C.; Calitz, F.J.; Fourie, P.H. Effects of adjuvants on deposition efficiency of fenhexamid sprays applied to Chardonnay grapevine foliage. Crop Prot. 2010, 29, 843-852.

28. Dorr, G.J.; Kempthorne, D.M.; Mayo, L.C.; Forster, W.A.; Zabkiewicz, J.A.; McCue, S.W.; Belward, J.A.; Turner, I.W.; Hanan, J. Towards a model of spray-canopy interactions: Interception, shatter, bounce and retention of droplets on horizontal leaves. Ecol. Model. 2014, 290, 94-101. [CrossRef]

29. Yuzugullu, O.; Erten, E.; Hajnsek, I. Rice Growth Monitoring by Means of X-Band Co-polar SAR: Feature Clustering and BBCH Scale. IEEE Geosci. Remote Sens. Lett. 2017, 12, 1218-1222. [CrossRef]

30. Fowkes, F.M. Acid-Base Interactions in Polymer Adhesion. Tribology 1978, 7, 119-137.

31. Jańczuk, B.; Białopiotrowicz, T.; Wójcik, W. The components of surface tension of liquids and their usefulness in determinations of surface free energy of solids. J. Colloid Interface Sci. 1989, 127, 59-66. [CrossRef]

32. Chen, J.R.; Wakida, T. Studies on the surface free energy and surface structure of PTFE film treated with low temperature plasma. J. Appl. Polym. Sci. 1997, 63, 1733-1739. 
33. Ramos, G.Q.; Ferreira, J.L.P.; Cotta, E.A.; Filho, H.D.D.F. Wettability and morphology of the leaf surface in cashew tree from the Amazon, Northern Brazil. Acta Sci. Biol. Sci. 2016, 38, 215-220. [CrossRef]

34. Wang, H.X.; Shi, H.; Li, Y.Y.; Wang, Y.H. The effects of leaf roughness, surface free energy and work of adhesion on leaf water drop adhesion. PLoS ONE 2014, 9, e107062. [CrossRef] [PubMed]

35. Fan, R.J.; Zhang, X.X.; Zhou, L.; Cao, C.; Du, F.P. Research on the wettability of peach leaf surfaces by OWRK method. Chin. J. Pestic. Sci. 2011, 13, 79-83.

36. Gao, Y.; Zhao, J.Y.; Shi, G.C.; Zhang, P.J.; Liu, Z.F.; Fan, R.J. Analysis of Leaf Surface Wettability for 4 Kinds of Industrial Crop Based on the Leaves Surface Free Energy and Component. J. Shanxi Agric. Sci. 2017, 45, 1331-1334.

37. Ren, L.Q.; Wang, S.J.; Tian, X.M.; Han, Z.W.; Yan, L.N.; Qiu, Z.M. Non-Smooth Morphologies of Typical Plant Leaf Surfaces and Their Anti-Adhesion Effects. J. Bionic Eng. 2007, 4, 33-40. [CrossRef]

38. Guo, Z.G.; Liu, W.M. Biomimic from the superhydrophobic plant leaves in nature: Binary structure and unitary structure. Plant Sci. 2007, 172, 1103-1112. [CrossRef]

39. Xue, X.Y. Application of Modern Pesticide Aerial Application Technology and the Impact on Rice Quality. Master's Thesis, Nanjing Agricultural University, Nanjing, China, 2013.

40. Wang, X.Q. Study on the Mechanisms of Ethylene in Regulating Brassica Napus Leaf Cuticular Waxes. Bachelor's Thesis, Southwest University, Chongqing, China, 2015.

(C) 2019 by the authors. Licensee MDPI, Basel, Switzerland. This article is an open access article distributed under the terms and conditions of the Creative Commons Attribution (CC BY) license (http://creativecommons.org/licenses/by/4.0/). 